\title{
Creating a COVID-19 bio-secure environment: Experience from a Medical Faculty in Sri Lanka
}

\author{
Dissanayake, A.S., Amarasena, S.D., \& Devasiri, V.
}

\begin{abstract}
The COVID-19 pandemic has presented educational institutions and educationists with unprecedented challenges with regard to the delivery of high-quality education. The challenges have been greater for the medical education institutions as the students engaging in clinical attachments work in a high-risk environment for COVID-19 transmission and place themselves and their associates at personal risk. The paper outlines the robust and pragmatic framework adopted by the Faculty of Medicine, University of Ruhuna to meet its twin goals of continuing to provide high-quality medical education whilst ensuring health and wellbeing of students. Measures were adopted to minimize exposure outside the educational environs as well as within the hospital and halls of residence. Guidelines were adopted, discussed and disseminated. Suitable personal protective equipment was defined and all students' inward settings requested to wear them. Unanticipated COVID-19 exposures took place on two occasions and the faculty risk assessment committee quarantined a few students with high-risk exposure within the halls of residence. The 'protected exposure' to minimize the risk of COVID-19 provided by the personal protective equipment they wore, prevented many students from being quarantined. All who were quarantined were COVID-RT-qPCR negative on day 14 of exposure. A community-acquired COVID-19 outbreak with 7 students testing positive for COVID-19 in the halls of residence required the Faculty of Medicine to institute the first civilian administered quarantine facility in Sri Lanka quarantining 200 students as first-line contacts and isolating a further 264 as second-line contacts. The administration of the quarantine facility was an unprecedented logistical challenge to the university administration and the academic staff. Meticulous attention to detail and commitment helped the faculty quarantine centre to meet up with all challenges and the 464 students all tested negative on the exit RT-qPCR. This was a zero cross-transmission rate for the infection and was a remarkable achievement for a quarantine facility. Leadership principles specifically aimed at managing crisis situations such as transparent communication, the urgency of action, commitment to core values and empowering all stakeholders formed the crux of the successful measures adopted. It is hoped that the wider lessons emanating from the experiences outlined in this paper will be of value to academic colleagues from similar medical educational settings to create bio-secure, COVID-19 protective environments in their respective institutions.
\end{abstract}

Keywords: COVID, Medical education, Risk minimization, Medical students, Quarantine, Isolation RTqPCR

\section{Introduction}

The COVID-19 pandemic has presented an unprecedented challenge to every aspect of human life. Interruptions to education both at school and university level have affected all societies, the world over. Educationists and educational policymakers have adopted innovative teaching methods to reach out to students who have had to be physically away

University of Ruhuna, Sri Lanka

Corresponding Author: Arosha S. Dissanayake, Department of Medicine, University of Ruhuna (80000), Sri Lanka

Email : arosha.dissanayake@gmail.com

DOI: http://doi.org/10.4038/seajme.v15i1.266 from teaching premises due to area lockdowns and physical distancing requirements. Despite these challenges, all educational institutions need to plan as much as possible, to complete their programmes without a delay in graduation, to ensure a steady stream of human resources for a country's development. This is especially important for schools of health/medicine as health workforce plays a key role in managing COVID-19 pandemic.

In addition to above difficulties which are common to all, medical faculties face a unique challenge as students perform their clinical training in a high-risk environment with potential exposure to a COVID-19 patient at any moment, in the course of their work. Hence 
medical faculty academic staff and administrators need to take leadership in astutely handling this crisis where they face competing interests. On one hand, they need to ensure good quality clinical training whilst securing the health and wellbeing of medical students. The fact that large numbers reside in the halls of residence, in close proximity to each other as one community confers on medical students, the inherent risk of being exposed to a rapidly spreading outbreak even if one of them were to become COVID-19 infected.

Sri Lankan COVID-19 epidemiology at present shows two peaks. Initially, in March 2020, there was a countrywide lockdown and all educational institutions were closed. Academic activities continued via internet platforms and the students were away from clinical appointments. The second peak occurred in October 2020 and keeping with government policy, only high-risk areas were locked-down and the rest of the country including medical faculties of universities continued to function. The case number in the second peak was much higher and was from many parts of the country compared to the first outbreak. Thus at the moment, there is an unprecedented risk of exposure of medical students to COVID-19 patients during clinical work and a COVID-19 outbreak in the student community. This paper outlines the robust and pragmatic strategies adopted and the leadership concepts followed in creating a COVID-19 bio-secure environment for medical students in the Faculty of Medicine, University of Ruhuna. It is hoped that disseminating this information would help other medical faculties both in the country and in the region to create similar bio-secure environments as well as to establish a platform to share experiences and learn from each other. Whilst sharing these measures we also focus on a leadership strategy for crisis situations. This leadership model could be adopted both by medical educational institutions as well as other organisations in the society for conducting their affairs well, whilst minimizing COVID-19 risk.

The Faculty of Medicine, University of Ruhuna was established in 1980. Presently it has a student population of approximately 1050 in six batches. Two batches are in the pre-clinical stage and four batches of students, numbering 620 in total, are engaged in clinical appointments in the Teaching Hospital Karapitiya. Altogether 650 students stay on-site within the medical faculty premises in the student halls of residence. The remaining 400 reside outside, within close proximity to the faculty.

\section{Methods and Strategies adopted}

On the $5^{\text {th }}$ of October 2020, the Sri Lankan government announced the detection of a fresh outbreak of COVID-19 after a lull of a few months. This initially happened in a garment factory and subsequently a popular fish market, both in Gampaha district of the Western Province of Sri Lanka. Several regions were detected to have COVID-19 infected persons and local area lockdowns were announced. Contrary to the expectation that the outbreak will be swiftly contained, it started spreading outwards. The faculty appointed a COVID-19 steering committee comprising of the Dean, the faculty medical officer, four student counsellors, the wardens of the male and female halls of residence within four days of the start of the second outbreak. It was decided to meet daily at noon in the office of the Dean to get updates on the situation and formulate measures to meet the challenges. As the number of students exceeded 1000, quick communication and conveying of crucial information was considered a problem and a mechanism was needed to obtain situational updates from students. Thus it was decided to invite the batch representatives and members of the medical faculty student union for these meetings to ensure more effective communication and relaying of information. Whenever it was deemed necessary, students from different batches were invited for discussions to the faculty auditorium which has a seating capacity of nearly 1000 and permitted the recommended social distancing. Whilst communicating in person continued, all relevant guidance documents were uploaded into student learning management systems (LMS). Communication channels had to be open not only to students but to fellow academic members of the staff as well as the clinicians and administrative staff from the Ministry of Health who were consultants in the hospital under whose guidance the students were engaged in clinical attachments. The Dean was to send regular email communications to the staff and the faculty clinical coordinator was requested to communicate with the hospital administration and clinicians. At all stages, the University of Ruhuna communicated the relevant information with the University Grants Commission which is the central administrative authority for all universities in Sri Lanka and the Ministry of Health under whose purview, all the hospitals operate. 
The principle of leadership in crisis management adopted here was communicating with transparency and communicating often (Edmondson, 2020). This helped to keep information flowing top to down, bottom to top as well as sideways. Transparent communication permitted us to be fully aware of both the obvious and the undercurrents regarding the prevailing uncertainty. The information we had access to, about the situation in the country as well as the Galle region was incomplete. The daily changing dynamics of the pandemic had us often playing a catching-up game. Despite incomplete information, every day we had to initiate urgent action, as complex situations evolved. These prompted us to adopt the following approaches.

At the beginning of the second outbreak, two potential exposures occurred. A friend of a medical student working in the affected garment factory visited him in Galle on Saturday 02.10.2020 when the first case in the second wave had still not been identified. Exposure was significant, for more than an hour. The friend underwent COVID-19 RTqPCR testing and was positive. This was communicated to us immediately by regional health authorities and the student was quarantined for 14 days, in his lodgings. His RT-qPCRs were negative. There was a religious function in a house which had been visited by some of the staff members of the faculty. An inmate of the house working in the same garment factory was positive for COVID19 RT-qPCR. The staff members were quarantined. They were tested with RT-qPCR and were negative. Hence the institution was not affected by the first stage of the second outbreak. The following measures were then adopted by the Faculty of Medicine COVID-19 steering committee.

1. All lectures were conducted via internet platforms. Laboratory sessions were conducted in duplicate thereby halving student numbers in one class.

2. There were several students confined to lock-down areas. Details were obtained from the student representatives, student counsellors and mentors. The lock-down area students were advised to confine themselves to homes. The area medical officer of health was contacted and updated on the details of the students. After 14 days of being confined to the home, they had RT-qPCR examinations and were permitted to return to student halls of residence and academic activities.

3. Soon after the lock-down commenced, a diarrhoea outbreak occurred in both male and female halls of residence. The previous practice of dealing with students who developed health complaints whist in the halls of residence was to refer them to the out-patients' department or a clinic in the Teaching Hospital Karapitiya, which is situated across the road from the faculty. As this process would entail unnecessary exposure of students to hospital patients, it was decided to form an Intermediate Healthcare Centre (IHC) for the faculty. It was established within the premises of one of the halls of residence. Basic facilities for medical consultation and some essential medicines were made available in the centre. The faculty medical officer, who is a specialist physician, attend to students with illnesses within the faculty premises itself. Approximately 80 students were afflicted by the gastroenteritis. Most were treated within the faculty. Two students too ill to be cared for in the IHC were referred to hospital for inpatient care. Respiratory symptoms were a frequent occurrence among the student community. They were assessed and the students whose symptoms were consistent with possible COVID-19 (Fever, cough and shortness of breath) were referred to the hospital outpatient department for RT-qPCR testing. Whilst the results were made available, the students were isolated from others in a separate room within the hall of residence.

4. Within two weeks, there were COVID-19 positive patients detected in the southern province and the numbers were escalating. In several hospitals from around the country, there emerged reports of hospital closures and quarantining of healthcare staff after exposure to COVID-19 patients. Thus it became gradually evident that sooner or later, students, in the course of their clinical appointments, would be accidentally exposed to a COVID-19 patient. The concept of 'Protected Exposure' was promoted to the students in addition to the current health guidelines to prevent transmission. After a review of scientific evidence, WHO and Ministry of Health guidelines, it was suggested to the students to wear personal protective 
equipment (PPE) during all clinical appointments (World Health Organization, 2020). All student batches were met and they were advised to wear a surgical mask, a face shield or goggles (all to prevent respiratory droplet transmission and splashing of secretions on mucous membranes), cloth or polythene overalls and disposable gloves (and use of alcohol-based sanitiser after each patient contact) or handwashing in the WHO recommended manner after each patient encounter to prevent contamination with respiratory secretions and thereby transmission of COVID-19. The PPE was to cost each student approximately Sri Lankan rupees 2,000 (approximately USD 11). While the large majority of students were able to afford the new PPE, 93 students among the 620 students engaging inward work were unable to finance it. We sought funding to purchase 200 such items of PPE to be distributed among this group of students. Prominent garment industries stepped in to provide PPE for students as part of their corporate social responsibility efforts. With all of the above in place, students who attend clinical attachments were requested to routinely wear these in order to convert unanticipated future COVID-19 exposures into 'protected exposures' with low risk of infection.

5. As anticipated the penny indeed dropped just one week later. A patient with chronic kidney disease on routine dialysis was admitted with shortness of breath to the emergency treatment unit and was subsequently transferred to the University Medical Unit. The patient's COVID RTqPCR test became positive. Within hours the faculty appointed a risk assessment committee comprising of the Dean, two specialist physicians and two microbiology specialists. All students who came into contact with the patients had a formal risk assessment by the committee, using the same criteria as laid down by the Ministry of Heath for the healthcare workers (Ministry of Health, 2020b). Medical students, by the nature of their work, become equal in risk to healthcare workers and forming a faculty-based risk assessment committee was an innovative action, necessitated by the urgency of circumstances.

6. Altogether 11 students were identified as having high-risk exposure due to not wearing appropriate PPE. Ten of the students who were in close proximity to the patient whilst on Non-Invasive Ventilation (NIV) which is termed an aerosol-generating procedure, without wearing a KN95 mask (they wore disposable surgical masks) were considered to have a high-risk exposure (Public Health England, 2020). The 11th student had been in contact with the patient for a period of more than one hour in the ward wearing only a surgical mask without a face-shield whilst the patient was not wearing a mask. He was determined to have a high-risk exposure by the risk assessment committee. The students were quarantined for 14 days since last exposure date, in a separate area within halls of residence as advised by the regional medical officer of health. They had an initial RT-qPCR which was negative in all students on day 7 of exposure (thereby providing some reassurance that their close contacts were safe from COVID-19) and had an exit RT-qPCR on day 14 which was also negative in all (Ministry of Health, 2020a).

7. Considering the lessons learnt from this incident, PPE guidance was updated and conveyed to students as verbal instructions as well as a document uploaded to the LMS. Common aerosolgenerating procedures were listed and students were advised to be in proximity of these only if they were wearing N95 masks or to keep a distance from these patients if they were wearing a surgical mask only.

8. Subsequently, another group of students came into contact with another COVID-19 patient in a surgical unit but as they were wearing appropriate PPEs, it was considered a low-risk exposure not needing any quarantine or RT-qPCR testing. The expectation was to remind and motivate all students to wear appropriate PPE all the time so that we may not need to quarantine any of them in the future.

9. The faculty needs to ensure not only the physical wellbeing of students but their psycho-social wellbeing as well. The COVID-19 related anxiety, stress and strife have interrupted the life of both students and academic staff, as never before. Student counsellors were at hand to support all students who were finding it 
difficult to cope. The Faculty Psychiatry department with specialist psychiatrists was available whenever the student counsellors felt that further support was necessary. This method had initially adopted after the first lockdown in June 2020 for the final year students awaiting their examinations. Motivating students to wear PPE presented challenges extending beyond the costs. Sri Lanka is a warm country and wearing a cloth or polythene overall for hours of ward work can be physically uncomfortable. Occasionally there were differing opinions on the need to wear these for all patient exposures but with regular communication, explanations and the fact that patients initially considered to be low risk subsequently being diagnosed as COVID-19 made detractors reconsider their perceptions. Presently it is standard practice that all students wear PPEs in hospital hours in order to make the unexpected exposure to a COVID-19 patient a protected ones conferring a low risk to the student.

The above actions highlight the second principle of leadership in crisis situations. There must be the urgency of action even when all the information required for decision making is lacking. Traditional leadership models suggest that all information is considered, analysed, several plans evaluated before taking the best possible decision. In a crisis situation, the scenario changes in a way that important decisions need to be taken and acted upon with urgency, within hours, as happened when establishing the medical student risk exposure assessment team.

The biggest crisis for us was still looming in the background. The principle of urgency of action was most crucially required when the crisis burst upon us at the least expected moment. With the personal protective measures described above, the Faculty of Medicine had ensured that no student contracted COVID-19 from the high- risk environment they worked in, the hospital wards. We recognised early that our Achilles heel was the possibility of a student getting a community infection whilst visiting home, returning to the halls of residence and then transmitting the infection to their close contacts in the hall or the clinical group resulting in a COVID-19 outbreak within the halls of residence. In anticipation of this eventuality, to minimize the spread within the student community, we had clustered the students to their clinical groups. There were approximately
15-20 students in each cluster. The epidemiological principal was if one of the students had a community-acquired COVID-19 infection, the first line contacts would be limited to the cluster and the need for home/institutional quarantine would be restricted to the cluster. The idea was that if the infection was restricted to one cluster of students who were residing, dining, studying and doing clinical work together, the infection would be easier to contain. As anticipated, a student who returned from visiting their residence had a COVID-19 RT-qPCR done performed for a complaint of anosmia and was detected to have COVID-19 infection. The remaining 14 students from the cluster were screened for COVID-19 with RT-qPCR testing and the second infected student was detected within the cluster. Whilst the infected students were transferred to treatment centres, a further 16 students from a related cluster were also tested and quarantined in a separate area in the halls of residence. Thus during the initial stage, 29 students were quarantined. The regional health authorities decided to screen the entire student body residing in the halls of residence and another 5 students with asymptomatic COVID-19 infection were detected from related student clusters. The authorities decided to quarantine all the students of the two batches considered as first-line contacts numbering 200. As second-line contacts a further 264 students from remaining batches needed to be isolated. The university and faculty administration had to take the most urgent of decisions, whether to send all students to their respective residences for home-quarantine or to convert the faculty halls of residence into a large quarantine facility. Considering the best interests of the students as well as the best interests of the country, to prevent dispersion of the COVID-19 infection to different parts of the country, it was decided to convert the halls of residences to a quarantine facility.

The challenges faced by the faculty academic staff in organizing the maintaining this quarantine facility were enormous. Most importantly we had to ensure there was no mixing of the two groups, those being quarantined and those in isolation to prevent cross-transmission of infection. Different areas within the halls of residence were arranged for the two groups. The faculty COVID-19 steering committee took the lead in ensuring the smooth running of the quarantine facility including the provision of food, medicines, other essentials, arranging for cleaning, garbage disposal and disinfection processes. Being confined to their own room for 14 days, away from families and 
academic work had taken a heavy psychological toll on the students. Members of the department of psychiatry, consisting of specialist psychiatrists and psychologists were assigned to care for the mental wellbeing of different batches of students and were in regular contact with the assigned students. $A$ communications team was appointed to speak regularly with the concerned students and guardians. On the 10th day of the quarantine, a musical session was conducted by the medical faculty academic staff for the students in an open area visible and audible to all the student halls of residences. The students joined in from their own rooms through windows and balconies without being in contact with others. This 'stress-busting' event did much to soothe the difficulties faced by all parties. After a 14day quarantine period, all 464 students tested negative in the exit RT-qPCR. Thus the University of Ruhuna became the first civilian administered quarantine facility in Sri Lanka to quarantine a vast number of first-line contacts (all other quarantine facilities are administered by the Ministry of Defense). The zero crosstransmission within the halls of residence was an achievement beyond expectations. As a recourse to the evolving challenge of COVID19 reaching the faculty premises from students returning from the community, student clustering efforts have been further strengthened and policies and protocols developed with contributions from the health authorities, academic staff and students themselves to minimize COVID-19 exposure from the community.

Uncertainty regarding the goals to achieve remains for us. Our vision is to prevent students from acquiring COVID-19 infection from the clinical setting while continuing the medical education to graduate them on time to fill the much needed health workforce needs and infusing an attitude of not turning away from pandemics, disasters when their services are much needed. The goalposts are shifting all the time and our actions are at times proactive, other times reactive. Though the end is not clear, the medical faculty has committed itself to a set of values. We wish to continue good quality undergraduate medical education whilst keeping the safety and wellbeing of medical student our foremost priority. Our actions will evolve by the day, as and when new needs arise. But our commitment to our values will not waver. This highlights the third principle in leadership in managing a crisis situation. We identify and commit to preserving what we value most. The leaders do not commit to the map but to the compass.
The faculty COVID-19 steering committee shared power with many stakeholders. The natural reaction of leadership in times of crisis is to tighten control. On the contrary, we shared power and sought expert opinions from academic staff, student counsellors, hospital administrators, hospital clinicians, regional health authorities and importantly from the students themselves. Initially, as there was a guideline issued by the Ministry of Health to close down universities, there was confusion with some concerned students and parents requesting an immediate and indefinite closure of the faculty till the pandemic was controlled. We have gradually empowered the students to protect themselves, protect each other, protect their families and loved ones too. This highlights the fourth principle of leadership in crisis situations. The way to move forward is to share power and empower individuals to play their roles in resolving the crisis.

It is important to continue medical education as the country needs medical professionals to address COVID-19 and other medical challenges. It is equally important to ensure that the quality of medical training is not compromised and the previous high standards continue to be met. The COVID-19 crisis has undoubtedly caused concern among both university academics as well as medical undergraduates about the risks and it is possible that the hands-on experience obtained in the clinical attachments is being compromised for safety's sake. That it is probably happening to an extent is undeniable. But the protective measures and the 'protected exposure' described and institutionalized in the Faculty of Medicine, as mentioned above has given much confidence to all parties that they can continue to engage in most of the hands-on training activities and only a few selected aerosol-generating procedures need extra caution. It was not easy to ascertain the full impact on the hands-on training of medical undergraduates. We plan to do a qualitative assessment of perceptions of both the medical undergraduates and the clinical teachers about the impact of COVID-19 on the hands-on clinical training programme. Methods for further improvement may come to light in this process. One objective way to assess would be to evaluate whether the marks obtained by the students facing COVID-19 restrictions are different from the marks of undergraduates for comparative clinical components from the preCOVID-19 period. Though marks obtained at clinical examinations are not truly representative of clinical exposure and 
experience, the present examination structure remains our mode of evaluation for the award of the MBBS degree. Only time will tell us the complete story.

\section{Conclusions}

The Faculty of Medicine, University of Ruhuna has been proactive in meeting the crisis presented by the COVID-19 pandemic to medical education. It has adopted measures consistent with leadership principles recognized not only in the educational sector but by the social and economic sectors too. We have communicated with transparency, communicated often and communicated with all stakeholders. We have acted with urgency to meet each contingency. We have committed ourselves steadfastly to the twin values of providing high standard medical undergraduate education as well as ensuring health, safety and wellbeing of the students. We have shared power with all stakeholders, including the students. It is still early in the day but we hope the continuation of these measures will help us move forward, meeting challenges successfully. In the process, the Faculty of Medicine became the first civilian administered quarantine facility in Sri Lanka and succeeded in having a zero cross-transmission rate within the facility. We believe sharing our experience will help similar organizations to adopt some of these successful strategies to counter the COVID-19 pandemic which has challenged most of the traditional approaches we have taken in the past, to achieving our desired goals and Gunasegaran, Mr Abisina Bujang Saili and Mr Sanjay Raj Gunaravi for their assistance in this project.

\section{Competing interests}

The authors declare that they have no financial, consultant, institutional or other conflicts of interest.

\section{Authors' contributions}

$A D, A S$ and $V D$ were all involved in planning and executing the above-mentioned measures and drafting of the manuscript

\section{Acknowledgements}

We thank members of the Faculty of Medicine, COVID-19 Steering committee for assistance in both planning and execution of measures to create a COVID-19 bio-secure environment.

\section{List of Abbreviations}

COVID-19: Corona Virus Disease-19

LMS: Learning Management System

RT-qPCR: Real Time-quantitative Polymerase Chain Reaction

PPE: Personal Protective Equipment

\section{References}

Edmondson, A. C. (2020) How to Lead in a Crisis, Video. Available at: https://www.hbs.edu/covid19-business-impact/Citations/How-to-Lead-in-aCrisis (Accessed: 18 November 2020).

Ministry of Health (2020a) Quarantine process to be followed by all immediate contacts of a COVID19 patient and any person entering into the country through ports of entry during the COVID19 pandemic situation. Colombo. Available at: http://www.epid.gov.lk/web/images/pdf/Circulars /Corona virus/Home Quarantine Guidelines.pd f.

Ministry of Health (2020b) Screening and management of healthcare workers following exposure to a confirmed/suspected case of COVID-19. Colombo. Available at: https://www.hpb.health.gov.lk/media/pdf/screeni ng-and-management.pdf.

Public Health England (2020) COVID-19 infection prevention and control guidance: aerosol generating procedures, Public Health England, Medical Procedures. Available at: https://www.gov.uk/government/publications/wu han-novel-coronavirus-infection-prevention-andcontrol/covid-19-infection-prevention-andcontrol-guidance-aerosol-generating-procedures (Accessed: 18 November 2020).

World Health Organization (2020) Rational use of personal protective equipment (PPE) for coronavirus disease (COVID-19): interim guidance, 19 March 2020. Geneva PP - Geneva: World Health Organization. doi: CC BY-NC-SA 3.0 IGO. 